\title{
On Hard and Soft Models to Analyze Trust Life Cycle for Mediating Collaboration
}

\author{
Simon S. Msanjila and Hamideh Afsarmanesh \\ Informatics Institute, University of Amsterdam, Science Park 107, \\ 1098 XG, Amsterdam, The Netherlands \\ \{s.s.msanjila,h.afsarmanesh\} @uva.nl
}

\begin{abstract}
Mediating short-term collaboration among organizations is very challenging in today's open world due to: the increasing intensity of market competition on acquisition of opportunities, the demand for the large amount of resources and the large number of different competencies, and the continuous increasing scarcity of resources, among others. One approach for organizations to reduce the severity of these challenges is joining their initiatives through collaborations. However, each organization has interests and goals that might be different and contradicting with those of other partners. Consequently, establishing fruitful collaboration is challenging and a proper approach to mediate collaboration among organizations is needed to support resolving emerging disputes during their interactions. Creating trust among organizations encourages them to quickly join their efforts to respond to these challenges and thus commit to the established collaboration. This paper addresses trust as a way to mediate collaboration among organizations. It addresses aspects related to softmodels and hard-models of trust and presents the application of these models in analyzing trust subjectively and objectively respectively. The paper finally introduces stages of life cycle of trust among organizations.
\end{abstract}

Keywords: Inter-organizational trust, trust life cycle, models of trust.

\section{Introduction}

Designing comprehensive trust models and mechanisms to address challenges related to mediating business oriented collaboration among organizations has become a fundamental focus of research on trust. Despite recent achievements from research addressing modeling of trust, there are still insufficient generic and customizable models, mechanisms and tools to support emerging requirements on trust analysis. Most available solutions supporting trust analysis are limited to: a specific application case (e.g. multiagent systems), known actors (organizations or individuals), or domain of study (e.g. health domain). These solutions are also limited to some specific set of trust criteria applied to their development.

Business collaborations among organizations are usually objective specific and shortterm in nature [5]. Developing bespoke solutions, such as models, mechanisms, tools, etc., to support analysis of trust among organizations for the purpose of mediating their collaboration is quite challenging. It is more challenging to analyze inter-organizational 
trust in virtual collaboration where organizations do not know each physically and interact through computer networks. A key catalyst to this challenge is the unclear picture of future business objectives that are usually dependent on market opportunities [4]. There is a need to develop customizable trust solutions to mediate collaboration among actors for every business objective.

This paper characterizes trust for mediating collaborations among organizations. It presents aspects of hard-models and soft-models of trust relationships and then applies these concepts in characterizing life cycle of trust among organizations.

\section{Nature and Dimension of Trust}

Nature of trust: The following are some characteristics of trust that have been identified for relationships between organizations:

\begin{tabular}{|l|l|}
\hline Nature aspect & Description \\
\hline Multi-level & $\begin{array}{l}\text { Trust level is not an absolute value that can be measured once and applied in all cases. } \\
\text { Each measured trust level depends on many factors such as involved organizations, } \\
\text { available data, purpose of the assessment of the trust level, set of trust criteria, etc. }\end{array}$ \\
\hline Multi-criteria & $\begin{array}{l}\text { Trust is addressed as a multi-criteria subject. Every case requiring trust establishment is } \\
\text { different and will need specific set of trust criteria to assess trust level of actors. }\end{array}$ \\
\hline Cultural-rooted & $\begin{array}{l}\text { Trust is closely tied to the norms, values and beliefs in the society. In addition to } \\
\text { trust objective, the cultural practices and believes may influence perceptions of trust } \\
\text { and preference on criteria that can be applied to assess trust level of organizations. }\end{array}$ \\
\hline $\begin{array}{l}\text { Communication } \\
\text { based }\end{array}$ & $\begin{array}{l}\text { Trust is the outcome of moral communication behaviors, such as providing accurate } \\
\text { information, demonstrating sincere and openness, etc. }\end{array}$ \\
\hline Dynamic & $\begin{array}{l}\text { Trust perception and preferences are not static rather they change depending on } \\
\text { different factors such as involved other organizations, goals of the collaboration, etc. }\end{array}$ \\
\hline $\begin{array}{l}\text { Multi } \\
\text { dimensional }\end{array}$ & $\begin{array}{l}\text { There are several dimensions of trust that characterize the dynamic nature of } \\
\text { trustworthiness of trustees as addressed below. }\end{array}$ \\
\hline
\end{tabular}

Dimension of trust: Dimension of trust refers to the characteristics which indicate the dynamic nature of trustworthiness. Dimensions of trust are operational aspects of trust that when properly addressed may contribute to enhance their trustworthiness of organizations. Following are some dimensions of trust:

\begin{tabular}{|l|l|}
\hline Dimension & Description \\
\hline Integrity & The belief that organizations are fair in all decision that are made through or by them. \\
\hline Reliability & The belief that organizations will do what they promise to do and act consistently. \\
\hline $\begin{array}{l}\text { Openness } \\
\text { and Honesty }\end{array}$ & $\begin{array}{l}\text { This dimension addresses the amount and accuracy of information that is shared among } \\
\text { organizations, and how sincerely and appropriately it is communicated to others. }\end{array}$ \\
\hline Vulnerability & $\begin{array}{l}\text { The organizations' willingness to participate in relationships and commit transactions. } \\
\text { Vulnerable is due to the belief that another organization is competent, open, honest, } \\
\text { concerned, reliable, and identified with common goals, norms, and values. }\end{array}$ \\
\hline $\begin{array}{l}\text { Popularity } \\
\text { branding }\end{array}$ & $\begin{array}{l}\text { Popularity and branding measure the extent to which organizations address common } \\
\text { goals, norms, values and beliefs associated with the involved society culture and values. } \\
\text { This dimension indicates how connected the actor feels to the (seen by the) society. }\end{array}$ \\
\hline $\begin{array}{l}\text { Influence } \\
\text { mutuality }\end{array}$ & $\begin{array}{l}\text { The degree to which organizations agree on who has rightful power to influence one } \\
\text { another. For the stable trust relationships the actors must be able to influence each other. }\end{array}$ \\
\hline Satisfaction & $\begin{array}{l}\text { The extent to which one organization feels favorably toward the other as expected in the } \\
\text { relationship. A satisfying relationship is one in which the benefits outweigh the costs. }\end{array}$ \\
\hline Commitment & $\begin{array}{l}\text { The extent to which one organization believes the trust relationship is worth to maintain } \\
\text { and promote. Two sub-dimensions of commitment are continuance: which refers to a } \\
\text { certain line of action, and affective: which is an emotional orientation. }\end{array}$ \\
\hline
\end{tabular}




\section{Inter-personal and Inter-organizational Trust}

As a subject, trust has gained increased attention and has been examined in both research and practice. It has been widely studied, most importantly as a component of relationships among organizations as well as among individuals. Below we describe and distinguish inter-personal and inter-organizational trust.

Inter-personal trust: Challenges related to inter-personal trust date far back and correspond with the beginnings of human life. Researchers have indicated that trust is important in smoothening inter-personal relationships [8]. Inter-personal trust has been practiced mostly in social relations and it is usually subjective in nature. Subjective trust is the most adopted and practiced form of trust for smoothening interactions among individuals. Subjective trust is created on the basis of qualitative data and is opinion-based. Some fundamental sources of information for creating subjective trust among individuals include experience and knowledge of trustors on trustees, recommendations of third parties on trustees, trustees' reputations, etc.

Inter-organizational trust: A key challenge on the establishment of collaboration among organizations is the selection of trustworthy partners for the purpose of fulfilling opportunities. Due to sensitivity nature of business goals the inter-organizational trust is rationally analyzed and supported with formal reasoning.

Traditionally, trust among organizations was only established "bi-laterally" and subjectively based on reputation and recommendation from others. In large collaborative networks [10], applying traditional approaches for creating bilateral trust among organizations is difficult, mostly due to the following reasons: (1) It is hardly feasible for trustors to collect reputation data or peer's opinions about the trustworthiness of trustees, with whom they had never interacted before. (2) It is hardly feasible to (rationally) reason on the trustworthiness of actors based on subjective data. Therefore, subjective trust (opinion-based) is too risky when applied alone and rational trust (fact-based) is required to be created among organizations to act as a foundation for evolution of trust during the goal oriented collaborations.

Rational trust is created on the basis of quantitative data and is fact-based. The main source of trust-related data is the organizational performance which is accumulated in the past from different activities in which it participated, both in collaboration with other partners, and as an individual organization. Rational approaches for assessing the level of trust in organizations employ formal mechanisms, such as mathematical equations, which in turn provide some formal reasoning of the resulting level of trust [11].

Inter-organizational trust vs. inter-personal trust: A fundamental difference between inter-personal trust and inter-organizational trust relate to their antecedents. Some antecedents of inter-organizational trust are known, such as the shared values, the previous interactions, and the practiced behaviors [10], and are aimed at preparing organizations towards trusting each other. As stated above inter-personal trust is very subjective in nature and dependent on individual's opinions. It is not yet clear what antecedents does an individual need to meet to be trusted by others.

Some other fundamental aspects applied in this paper to distinguish between interpersonal trust and inter-organizational trust include: trust criteria, models of trust relationships, and mechanisms for assessing trust level as addressed below. 
- Trust criteria: One important aspect of characterizing trust is the identification of trust criteria for various actors. Trust criteria for organizations are fact-based in nature and in our research are characterized to express organizational performance data [11]. Inter-personal trust is measured based on opinions, rating, voting and other types of subjective or probabilistic measures [13].

- Models of trust relationships: Considering the key role trust plays in mediating collaboration among actors, an understanding of concepts relating to inter-actor trust relationships must be properly addressed. In [12] we examined the need of modeling trust relationships between organizations. Models of trust relationships between organizations are hard in nature applying fact-based elements, e.g. those related to their performance. Models of trust relationships between individuals are soft in nature and they represent subjective data related to trust (Section 4).

- Mechanisms for assessing trust level: As discussed earlier, inter-personal trust is mostly regarded as a subjective aspect and its measurements have been frequently based on the probability perceived by a trustor that a trustee can do something [6]. However, a probability-based assessment works well when trust is regarded as a subjective aspect. It is easier to count opinions that supported the positive reputation of trustees and thus use these counts to calculate their trustworthiness as probability values. In such practices, the need to formally reason about results of the assessment is not necessary. Today, rational mechanisms for assessing the level of trust in organizations are needed to support making formal reasoning on the results. Assessing level of trust in organizations is fundamental to successfully establishing their trust relationships. Designing rational (fact-based) mechanisms for assessing the level of trust in organizations is of particular importance to enhance accuracy of computed trust level. In [10] we proposed a mathematical approach for formulating mechanisms to assess the trust level of an organization. Thus mechanisms are formal and support rational reasoning on the results.

\section{Soft Models and Hard Models of Trust}

Development of models of trust has attracted concentrated efforts from researchers and many variants of trust models have been developed. As these proposed models are built on different underlying concepts, a need is evident for systematic approach to categorize, evaluate and improve these models in order to apply them effectively.

We categorize models of trust as hard-models, soft-models and semi-soft-models. Hard-models of trust are designed to support the management of trust among organizations on basis of fact-based data, such as their performance data. Soft-models of trust are designed to support the management of trust among organizations on the basis of subjective data such as reputation, opinions, etc. Semi-soft-models of trust capture some aspects of both rational and subjective trust analyzes.

Hard-models of trust have been developed and applied to support management of trust in some specific cases. In research addressing security of distributed systems, hard-models of trust are developed to support the management of trust among communicating systems. These models can only detect and analyze trust based on data delivered through crypto-based mechanisms [9]. Furthermore, in security studies of systems, hard-models of trust are applied to develop mechanisms that can be used to 
analyze trust applying data related to violations of security assumptions, security performances, vulnerability, etc. In our previous work on inter-organizational trust we have proposed hard-models of trust based on mathematical equations [11]. Models are applied to formulate mechanisms for assessing the level of trust in organizations considering measurable criteria and a formal reasoning on results is supported.

Therefore, a number of different kinds of hard-models of trust are already developed for specific application cases. Hard-models do not consider the aspects of all stages of trust life cycle which is characterized with some soft aspects of trust as further addressed in Section 6. Thus there is a lack of conceptualized hard-models of trust that are comprehensive enough to adequately apply in any emerging objectives and can be customized to meet interests of any kind of organizations. Although hardmodels of trust provide a base or foundation for designing other models for higher stages which consists of qualitative elements (soft-models of trust) there is a need for looking into the possibility of integrating the two types of models.

Soft-models of trust capture the trust relationships between organizations that are based on observable evidences about trustee's behavior, either through direct experience (witness reputation) or indirect experience (certified reputation). Hence softmodels of trust use social control mechanisms, namely, based on how actors socially recommend each other. Analysis approaches for soft-built trust include: probability measurements based on positive and negative opinions, logical operations to analyze trust transitivity, prediction analysis in relation to risks, etc. Existing models of trust for inter-personal interactions can be classified as soft-models [7].

While each type of models is able to provide a different focus on addressing trust issues in collaborative networks, both types also come with some drawbacks. Hardmodels of trust are difficult to apply in real life as they demand a complete set of data for all considered criteria. Soft-models of trust suffer from the lack of traceability and the problem of trust saturation which can leave actors vulnerable to cheat attacks.

Some attempts have been made by researchers to develop models which can capture a few aspects of both had-models and soft-models of trust, here referred to as semi-soft-models of trust. A causal model as inspired in the discipline of systems engineering supports analysis of causal influence among measurable factors (hardmodel aspects) but allows some qualitative reasoning to be made on the nature of influences (soft-model aspects). For example, as shown in Fig. 1 while factors "cash capital" and "capital" are both measured quantitatively with numbers the influence of cash capital on capital is qualitatively assumed as positive.

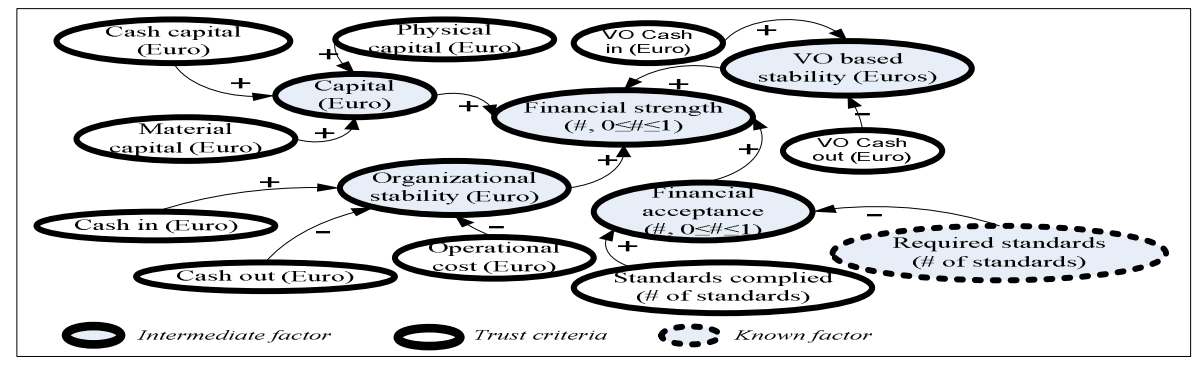

Fig. 1. Semi-soft-model of trust for the organizational economical perspective 
Causal models as semi-soft-models of trust can also be transformed to hard-models of trust in form of mathematical equations. On the basis of assumptions as inspired by the discipline of systems engineering the formulation of mathematical equations (hardmodels of trust) applying causal models (semi-soft-models of trust) is thoroughly addressed in [11]. The formulated equations are then applied in designing mechanisms for assessing the level of trust in an organization. For example, the equations for capital (CA) and financial acceptance (FA) can be represented as follows [11]:

$$
C A=C C+P C+M C \text { and } F A=\frac{S C}{R S}
$$

Where CC represents cash capital, $P C$ represents physical capita, $M C$ represents material capital, SC represents standards complied, and $R S$ represents required standards.

\section{Mediating Organizations' Collaboration}

The term mediation is applied in various disciplines carrying different meaning but what is common is the facilitative role it plays. In law, mediation refers to a form of "alternative dispute resolution" or "appropriate dispute resolution", which aims to assist two (or more) disputants in reaching an agreement [3]. As practiced in law, several different styles of mediation exist such as evaluative, facilitative, and transformative. Evaluative mediation does have somewhat of an advisory role in that its practitioners evaluate the strengths and weaknesses of each side's arguments should they go to court. Facilitative mediation acts as an interface to facilitate the flow and exchange of information for the disputants and therefore, enhance their communication. Transformative mediation looks at conflict as a crisis in communication and seeks to help resolve the conflict thereby allowing people to feel empowered in themselves and better about each other. In computer science, mediation is viewed as a facilitative instrument supporting the flow of information among nodes through the use of computer networks. It is an information request answered by providers, such as search engines, based on the analysis of the content of each information objects within a data collection such as a digital library, WWW, etc. [14].

Mediating collaboration among organizations needs to be looked at considering a variety of aspects from which a number of different alternatives can be preferred. Commonly, in business practice, contracts have been used as a fundamental approach to mediate and support collaboration among organizations. Contracts can be made between the collaborating partners (direct contracts) or through a third party (insured contracts). Contracts consist of "get out" clause such as penalties. Organizations sometimes end their commitment when they feel that possible risks are higher than expected gains and thus it is worthy to pay the penalties and quit the collaboration. Creating trust among organizations to mediate their collaboration has emerged as a potential approach to enhance commitment in the network towards achieving the common goals. Trusted organizations feel the burden of meeting their promises to sustain their trust due to the fact that trust cannot be traded.

Creating trust among organizations to mediate their collaboration focuses on avoiding possible future disputes related to their interactions by ensuring that each involved partner possesses the acceptable trust level. When the trust level of all involved partners is above the threshold, there is a high chance that their collaboration will be 
smooth and effective. The evaluation of trust level of partners is objectively performed and the computation applies mechanisms implemented based on hard-models of trust. As such, the avoidance of future disputes among organizations by rationally evaluating their trust level corresponds to the evaluative role of mediation as inspired in the discipline of law.

However, if disputes among organizations occur during their collaboration, such as those related to conflicts caused by issues like the lack of commitment, the failure to achieve promised goals, etc., then promoting and enhancing trust among partners can be applied to resolve such disputes. As such, the role supported by promoting and enhancing trust among organizations is similar to the two aspects of mediation -the facilitative and the transformative - inspired in law and/or computer science. The first aspect is related to finding proper channels to act as interface between organizations to exchange the information needed to sustain their trust (facilitative mediation). The second aspect is related to supporting each organization to decide on suitable information to communicate to other organizations (transformative mediation).

Therefore, management of trust to mediate collaboration among organizations must address the three fundamental aspects of mediation of: (1) Evaluative: Support the measurement of trust level of organizations, (2) Facilitative: Support the provision of information for creation of trust among organizations and establishment of their trust relationships, and (3) Transformative: Provide methodologies to maintain and sustain trust among organizations during the collaboration.

\section{Stages of Trust Life Cycle}

Trust between organizations evolves (grow, remain uniform or deteriorate) with time from its birth (creations) and keeps evolving with time while organizations know, learn and experience each other through collaboration. Also, trust among organizations and in particular, those aiming at achieving common goals through collaboration, has been observed as objective specific and need to be built on the foundation of fact-based data and thus must start with a rational analysis. With time trust of organizations will evolve to incorporate opinion-based analysis applying recommendations and reputation data (soft-built trust) (See Fig. 2).

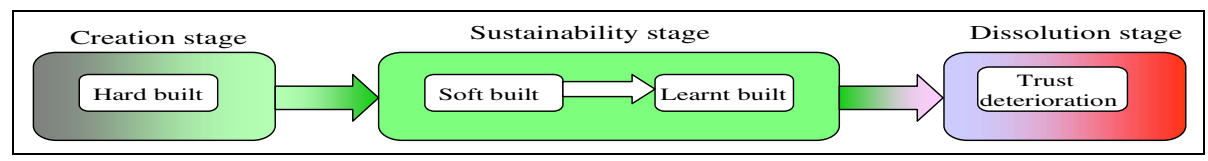

Fig. 2. Trust life cycle stages

And with relatively long-time some learning based analyzes of trust will also be incorporated in the trust life. Thus trust analysis needs to consider the notion of time and must address the evolution of trust level of organizations. Previous studies have indicated that requirements for collaboration among organizations differ depending on the life stage of the collaborative networks [1]. It has been shown that collaborative networks undergo three life stages namely: creation, operation and dissolution or 
metamorphosis [2]. Since trust among organizations is created to smoothen their collaboration we have characterized trust life cycle in three stages similarly to those of collaborative networks, namely: creation, sustainability and dissolution.

\subsection{Creation Stage}

This stage is initiated when organizations which do not know or know little about each other realize the need for trusting each other and thus start looking for fact-based data to analyze trust of others. The stage is experienced once, and in particular for those organizations that have never collaborated in the past. Thus to create trust among organizations, comprehensive rational data is needed and must be thoroughly analyzed which leads to the so-called "hard built trust". Only hard-built trust can be realized at this stage and acts as foundation for other next stages. The measurement of trust level, in this stage, reflects the evaluative aspect of mediation for collaboration.

Hard-built trust is established based on solid and measurable data. Formal mechanisms are needed to both measure the trust level and support reasoning on the computed results [11]. Hard-models of trust need to be developed to support establishment of hard-built trust. Using these models, it is possible to develop potential mechanisms and services to support processes related to trust management, such as assessment of the levels of trust in organizations [12].

Considering today's businesses that need organizations to virtually collaborate to serve a single customer, the inter-organizational trust creation must be based on facts. Measurable trust criteria and formal mechanisms must be applied to ensure that the analysis results are as accurate as possible and can be supported with some formal reasoning [11]. Therefore, the next stage - the sustainability stage - of trust life cycle should be initiated only when the involved organizations have proved beyond reasonable doubt that they have met the base trust level which is assessed based on mechanisms established using hard-models of trust.

\subsection{Sustainability Stage}

Sustainability stage inherits the success of the trust creation stage. This stage starts when organizations are convinced with provided rational data related to trust. Thus, as shown in Fig. 3, the computed trust level of each organization is either equal or higher than the base trust level. In this stage of trust life cycle, organizations focus on enhancing the trust to each other through looking at everyone's behavior (soft trust) and learning their achievements with time (learnt-trust). This stage has two sub-stages: "soft-built trust" and "learnt-built trust".

Soft built trust is created based on semi-rational data or subjective data, mostly captured from the analysis of behavior and initiatives of organizations during the post "hard built trust". At this sub-stage, organizations are convinced with the provided fact-based data, but they want to softly analyze responses and behaviors of others when hard-built trust is put into practice. This kind of trust is experienced when organizations want to learn about others and predict their possible behavior or commitment in near future.

Learnt-built trust is achieved through relatively long period of collaboration which is enough to thoroughly learn the activeness and long-time achievements of trustees. 
This sub-stage is mostly focused on learning about trustees and trying to compare their achievements to the expectation which was predicted during the soft-built trust establishment. While collaborating, a number of changes, such as market conditions, might influence the behavior of organizations. Thus learnt-built trust allows the trustors to analyze the response of trustees to such changes. In this case, some sort of softbuilt trust might again occur to re-predict possible future behavior. The sustainability stage incorporates both the facilitative and transformative aspects of mediation of collaboration. The establishment of soft-built trust and learnt-built trust which is based on the intensity of observation on organizations reflects the facilitative aspect of the mediation of collaboration. The support for and analysis of evolution of trust of organizations during the sustainability stage reflects the transformative aspect of the mediation of collaboration. Sustainability of trust can occur following one of the four disjoint possible scenarios of evolution of trust level as shown in Fig. 3.

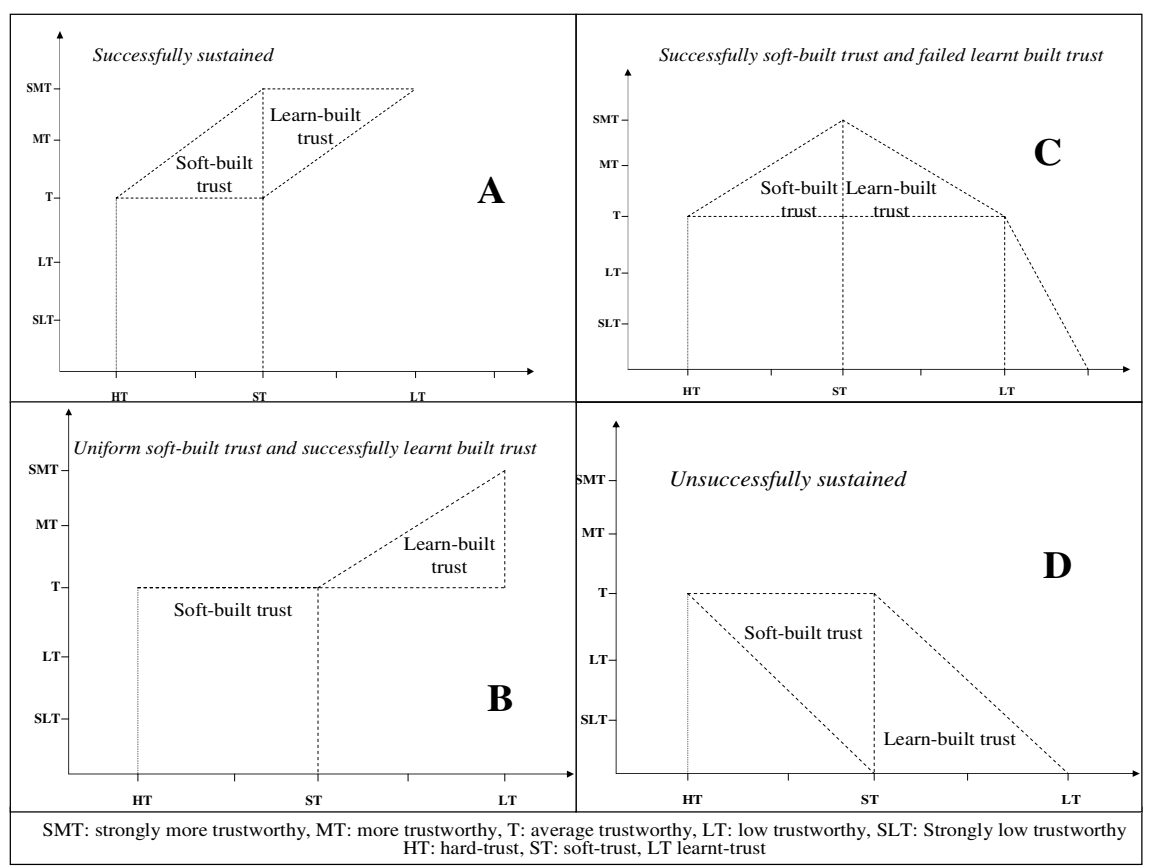

Fig. 3. Scenarios of trust evolution during the sustainability stage

Scenario A - Trust between organizations is successfully sustained: In this scenario, the level of trust in organizations keeps growing during the entire sustainability stage. This scenario occurs when the hard-built trust is realized and the trust level of organizations keeps on growing during the soft-built trust sub-stage. Thus at this point in time the organizations are convinced on the validity of hard-built trust and the positive predicted behavior of organizations is correct which means they can start collaborations. Furthermore, the trust level of organizations also keeps growing during the learnt-built trust sub-stage and thus it continues becoming stronger due to the positive 
results from the organizations' learning of each other. This is experienced, when organizations are effectively performing collaborative activities and are optimally achieving compatible/common goals. Thus organizations' experience and learning are positive, and they enhance trust in each other.

Scenario B - Uniform soft-built trust and successfully learnt-built trust: In this scenario the trust level of organizations remains uniform during the soft-built trust sub-stage, but it grows during the learnt-built trust sub-stage. Thus the same situation as explained in scenario " $\mathrm{A}$ " for the learnt-built trust sub-stage is experienced here.

Scenario C - Successfully soft-built trust and failed learnt-built trust: This scenario may occur when the trust level of organizations keeps growing during the soft-built trust sub-stage as explained in scenario "A" above. However, as the transition to learnt-built trust sub-stage starts, some failures emerge (such as failure to show the promised competencies) which lead to insufficient commitment of organizations in collaborations. Consequently, trust starts to deteriorate due to negative results from the learning process. This situation leads to dissolution stage.

Scenario D - Unsuccessfully sustained: This scenario may occur when organizations fail to realize soft-built trust. It may occur, for example, when organizations' behavior does not match with their trust level which was computed during the trust creation stage by using fact-data (hard-built trust). For instance, although organizations have strong trustworthiness, they can show poor behavior and indicated lack of proper initiatives to realize collaborative goals. In particular, this situation can occur when organizations indicated by using their fact-based data that they are capable of doing something but failed to prove during the negotiation, such as failing to show evidence of validity of their data trust related. Sometimes, trust among organizations may remain unchanged during the soft-built trust sub-stage. However, during the transition to learnt-built trust sub-stage some failures might be immediately experienced (such as failure to show the promised competencies). For example, if inadequate initiative is observed during the learning sub-stage and negatively affect the possible future collaboration then levels of trust in organizations may start to deteriorate. If the deterioration of trust continues to the point that the levels of trust in organizations become less than the base trust level then the dissolution stage starts.

\subsection{Dissolution Stage}

This stage occurs when organizations cannot trust each other enough to continue collaborating. Depending on the intensity of collaboration achieved in the past, during the dissolution stage a number of consequences might need to be inherited or divided between them. For example, distributing between them the incurred loss or achieved profit. In some cases a third party might be involved to smoothen the dissolution process, such as acting as an insurer to both conflicting parties. In this case, assets that cannot be distributed among the organizations, such as accumulated knowledge, can be transferred to the third party where all organizations can have fair access, compensation, etc. At the end of this stage, organizations terminate their collaboration and starts searching for new partners or looking for other options. As it is practiced in daily life, during the dissolution stage the fault trustees might attempt to re-create their trust to their trustors. This might include, for example, the provision of some 
new fact-based data or reputation data. The provision of the data by the fault trustees aims at persuading the trustors to reconsider or recomputed their trustworthiness. As such, if accepted by the trustors then the mechanisms discussed in the creation phase shall be used for manipulation of fact-based data. If only reputation data is available then the trustor organization might reconsider using the mechanisms discussed in sustainability stage but if and only if the trustor is convinced on the trustee's hardbuilt trust.

\subsection{Similarities and Differences between Trust Aspects in the Three Stages}

The base characteristic of trust during the creation stage (hard-built trust) is the rational nature. The analysis is based on fact-data and applies rational mechanisms and approaches. As such, measurable trust criteria are used and some automated systems as well as services are needed to support the required computations. The characteristics of rational trust are summarized in Fig. 4.

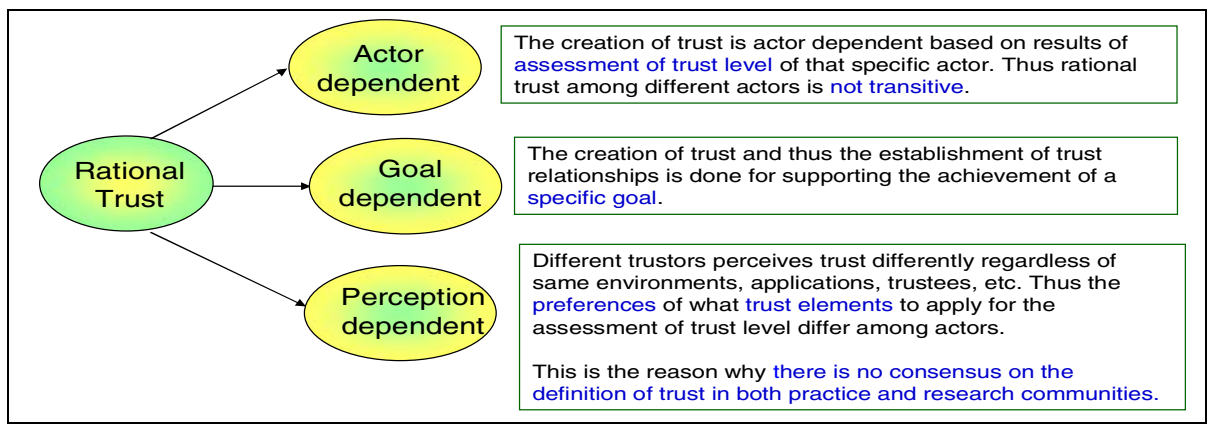

Fig. 4. Base concepts of rational trust among actors

The base characteristic of trust during the sustainability stage is its evolution with time. When soft-built trust and learnt-built trust are realized trust during this stage becomes transitive when applied to the similar applications or tasks. This means since the hard-built trust was realized during the creation stage, such as during the establishment and initiation of their trust relationships based on the results of the rational assessment of their trust level, and thus each organization can softly trust another organization based on the opinions or acquired reputation of other organizations. Opinions may refer to how trustees have been behaving in the previous interactions and how possible the trust in the trustees might evolve in the future.

\section{Conclusion}

Collaboration involves the mutual engagement of organizations to together address a challenge such as acquiring and responding to a business opportunity, which requires commitment, and it takes time, effort, and dedication. For this to be realized, trust among organizations needs to be properly managed to mediate their collaboration 
during the entire period. In this paper, we have addressed the characterization of aspects related to hard-models and soft-models of trust among organizations. We have applied these concepts to introduce and describe the life cycle of trust among organizations. We have also presented the application of trust in mediating collaboration among organizations.

\section{References}

1. Smith, T.F., Waterman, M.S.: Identification of Common Molecular Subsequences. J. Mol. Biol. 147, 195-197 (1981)

2. Afsarmanesh, H., Camarinha-Matos, L.M.: On the classification and management of virtual organization breeding environments. The International Journal of Information Technology and Management - IJITM 8(1), 1741-5179 (2009)

3. Afsarmanesh, H., Camarinha-Matos, L.M.: A framework for management of virtual organizations breeding environments. In: Proceedings of 6th PRO-VE 2005 - Collaborative Networks and their Breeding Environments, Spain, pp. 35-48. Springer, Heidelberg (2005)

4. Billikopf, G.: Party-direct mediation: Helping others resolve differences, 2nd edn. University of California (2009)

5. Camarinha-Matos, L.M., Afsarmanesh, H.: Collaborative networks in industry and services: research scope and challenges. In: COA 2007 - 8th IFAC Symposium on CostOriented Automation, Habana, Cuba, vol. 8, part 1, p. 10 (1-16) (2007)

6. Camarinha-Matos, L.M., Afsarmanesh, H.: Collaborative networks: Value creation in a knowledge society. In: Proceedings of PROLAMAT 2006, IFIP International Conference on Knowledge Enterprise - New Challenges. Springer, China (2006)

7. Gambetta, D.: Trust: Making and Breaking Cooperative Relations. Basil Blackwell, Malden (1988)

8. Grandison, T., Sloman, M.: A survey of trust in internet applications. IEEE Communication Surveys, fourth quarter (2000)

9. Gulati, R., Singh, H.: The architecture of cooperation: managing coordination costs and appropriation concerns in strategic alliances. Admin. Science Quarterly 43, 781-814 (1998)

10. Lin, C., Varadharajan, V.: A Hybrid Trust Model for Enhancing Security in Distributed Systems. In: Proceedings of International conference on availability, reliability and security (ARES 2007), Vienna, pp. 35-42 (2007) ISBN 0-7695-2775-2

11. Msanjila, S.S., Afsarmanesh, H.: A Multi-Model Approach to Analyze Inter-organizational Trust. In: Collaborative Networks reference modeling, pp. 195-216. Springer, New York (2008)

12. Msanjila, S.S., Afsarmanesh, H.: Trust Analysis and Assessment in Virtual Organizations Breeding Environments. The International Journal of Production Research, 1253-1295 (2007) ISBN 0020-7543

13. Msanjila, S.S., Afsarmanesh, H.: Modeling trust relationships in Collaborative Networked Organizations. International Journal of Technology Transfer and Commercialization 6(1), 40-55 (2007)

14. Mezgar, I.: Trust building for enhancing collaboration in VOs. Network-Centric Collaboration and Supporting Frameworks, 173-180 (2006)

15. Neuhold, E., Niederee, C., Frommholz, I., Stewart, A., Mehta, B.: The role of context for information mediation. In: The proceeding of International Workshop on Ubiquitous Data Management, Tokyo, Japan, pp. 3-5 (2005) 